\title{
A loop matters for FTO substrate selection
}

\author{
Zhifu Han ${ }^{1}$, Ning Huang ${ }^{1}$, Tianhui Niu ${ }^{1}$, Jijie Chai $^{1,2} \bowtie$ \\ ${ }^{1}$ National Institute of Biological Sciences, No. 7 Science Park Road, Beijing 102206, China \\ ${ }^{2}$ College of Biological Sciences and Biotechnology, Tsinghua University, Beijing 100084, China \\ $\triangle$ Correspondence: chaijijie@nibs.ac.cn \\ Received June 9, 2010 Accepted June 16, 2010
}

\begin{abstract}
Recent studies have unequivocally established the link between FTO and obesity. FTO was biochemically shown to belong to the AlkB-like family DNA/RNA demethylase. However, FTO differs from other AlkB members in that it has unique substrate specificity and contains an extended C-terminus with unknown functions. Insight into the substrate selection mechanism and a functional clue to the C-terminus of FTO were gained from recent structural and biochemical studies. These data would be valuable to design FTO-specific inhibitors that can be potentially translated into therapeutic agents for treatment of obesity or obesity-related diseases.
\end{abstract}

\section{FTO AND OBESITY}

Obesity poses a serious public health problem in the world. It is a leading cause of various common diseases including type 2 diabetes, cancer and heart disease (Abelson and Kennedy, 2004). Unfortunately, identification of genetic variations related to obesity had been unfruitful for a long time, although many efforts were made to this end. The reason for this may be that genetic screen is complicated by contribution of excessive caloric intake as well as a lack of physical activity to obesity (Loos and Bouchard, 2008). However, this situation has been changed with the application of genome-wide association studies (GWAS). In 2007, two groups (Dina et al., 2007; Frayling et al., 2007) independently found that a common variant (rs9939609) in the FTO (fat mass and obesity associated) gene is associated with Body Mass Index (BMI). People carrying variants in the FTO gene are more susceptible to obesity. Many other groups have subsequently confirmed such a correlation among individuals of different cohorts (Hinney et al., 2007; Ohashi et al., 2007; Scuteri et al., 2007). FTO locates on chromosome 16 in human and on chromosome 8 in mouse. Both the FTOs contain 9 exons while rs9939609 lies in the first intron (Groop, 2007).

Further evidence supporting the strong association of the FTO gene with obesity came from animal model studies. FTO-deficient mice with an altered or compromised FTO function are protected from high fat diet-induced obesity by affecting the whole body metabolism and regulating energy homeostasis (Fischer et al., 2009). A later investigation demonstrated that a dominant missense mutation (I367F) in the mouse FTO gene generated similar phenotypes to the knockout mice: reduced fat mass, increased energy expenditure and unchanged physical activity (Church et al., 2009). These two studies not only provide strong evidence for the GWAS data, but also establish a mechanistic link between FTO and obesity. Recent accumulation of data, however, suggests that a higher risk of obesity caused by FTO variants in humans is related to increased food intake and reduced satiety (Cecil et al., 2008; Kring et al., 2008; Speakman et al., 2008; Timpson et al., 2008; Wardle et al., 2008; Haupt et al., 2009). It is unclear why the mechanisms underlying FTOregulated obesity are different between mice and humans. Perhaps these data can be reconciled through studies on the molecular mechanism of how FTO is linked to obesity.

\section{ALKB-FAMILY DNA/RNA DEMETHYLASE AND SUBSTRATE SELECTION MECHANISM}

$\mathrm{Fe}(\mathrm{II}) / 2-$ oxoglutarate (2-OG)-dependent oxygenases play important roles in diverse biologic processes including collagen modifications, carnitine biosynthesis, phytanoylCoA metabolism, demethylation of methylated DNA and Histone lysyl and arginyl demethylation(Loenarz and Schofield, 2008). AlkB is a member of the $\mathrm{Fe}(\mathrm{II}) / 2-\mathrm{OG}-$ dependent oxidative DNA/RNA demethylases (Falnes et al., 2002; Aas et al., 2003). Eight $A / k B$ homologs are predicted to exist in human (Kurowski et al., 2003). Removal of the methyl group from 1-methyladenine (1-meA) and 3-methylcytosine (3meC) through oxidation by AlkB family protein has been 
experimentally demonstrated for E. coli AlkB (Falnes et al., 2002) and its human homologs hABH1 (Westbye et al., 2008; Müller et al., 2010), hABH2 (Duncan et al., 2002; Aas et al., 2003) and hABH3 (Duncan et al., 2002). In addition to DNA, RNA can also act as substrates of some AlkB members like AlkB, hABH1 and hABH3 (Aas et al., 2003; Westbye et al., 2008). The significance of RNA demethylation by AlkB has been demonstrated in vivo (Aas et al., 2003; Ougland et al., 2004). In contrast, knockout of AlkB members in mice did not generate striking phenotypes (Ringvoll et al., 2006). Therefore the biologic significance of DNA/RNA demethylation by mammalian AlkB homologs is still not well understood. At the cellular level, hABH2 was shown to have a primary role in guarding mammalian genomes against 1-meA damage (Ringvoll et al., 2006), whereas hABH3 may alleviate the deleterious effects of nuclear DNA/RNA methylation by repairing single-stranded DNA/RNA (ssDNA/RNA) lesions (Aas et al., 2003).

Biochemical studies have revealed the substrate specificity of AlkB family demethylases. AlkB, hABH1, hABH2 and hABH3 have a similar specificity for 1-meA and 3-meC, but they exibit different selectivity for ss or double-stranded (ds) DNA/RNA substrates. $\mathrm{hABH} 2$ was found to be strikingly more active on dsDNA than on ssDNA (Duncan et al., 2002; Aas et al., 2003). By contrast, hABH1 and hABH3 strongly prefer ssDNA/RNA as substrates (Duncan et al., 2002; Aas et al., 2003; Westbye et al., 2008), whereas AlkB has a slightly higher activity toward ss than ds nucleic acids (Falnes et al., 2002).

So far, several crystal structures of AlkB members, including AlkB, hABH2 and hABH3, have been solved (Sundheim et al., 2006; Yu et al., 2006; Yang et al., 2008). As expected, all the available structures reveal a conserved double-stranded beta-helix (DSBH) fold, termed as jelly-roll motif that coordinates a catalytically active iron center composed of a conserved His-Xaa-Asp/Glu-Xaa-His motif (Hausinger, 2004). Despite their highly conserved structures, different AlkB members exhibit distinct substrate specificities as mentioned above. Structural studies of AlkB and hABH2 in complex with dsDNA have provided insights into the mechanisms underlying substrate selection of the AlkB-family proteins. The structure of AlkB-dsDNA reveals that AlkB interacts with dsDNA exclusively through making contacts with the damaged strand (Yang et al., 2008). Squeezing the two bases flanking the flipped-out one was proposed to be responsible for base-flipping in the substrates. These observations provide an explanation for the preference of AlkB for ssDNA lesions over dsDNA ones. Compared to AlkB, $\mathrm{hABH} 2$ makes extensive interactions with both strands of the duplex DNA (Yang et al., 2008). Interactions between the complementary strand of dsDNA with hABH2 are mainly mediated by the nonconserved motifs in $\mathrm{hABH} 2$. In particular, Phe102 in hABH2 not conserved in $\mathrm{hABH} 3$ was proposed to play an important role for the preference of $\mathrm{hABH} 2$ for dsDNA (Yang et al., 2008).

\section{FTO BELONGS TO AN ALKB-LIKE DNA/RNA DEMETHYLASE}

Association between the FTO intron polymorphism and obesity prompted people to investigate the function of $F T O$ gene. Biochemical identification of FTO benefited from bioinformatics studies. By using profile-to-sequence and profile-to-profile based comparisons, Sanchez-Pulido et al. (2007) found that the $\mathrm{N}$ terminus of FTO protein possesses limited sequence similarity to the AlkB members. Intriguingly, the prediction revealed that FTO homologs exist in green algae Ostreococcus and diatoms but not in insects, worms or fungi. Understandably, the predicted structure of FTO protein contains a DSBH fold homologous to those of $\mathrm{Fe}$ (II) and 2-OG oxygenases. The four conserved residues His231, Asp233, His307 and Arg316, are predicted to be responsible for coordinating $\mathrm{Fe}(\mathrm{II}) / 2-\mathrm{OG}$. The bioinformatics studies were experimentally confirmed by in vitro biochemical assays. To identify the in vitro substrates of FTO, Gerken et al. (2007) tested many of the known substrates of human 2-OG oxygenases. Only the 3-methylthymine (3-meT) oligonucleotide was found to significantly stimulate turnover of 2-OG. In contrast, the oligonucleotide containing 1-meA, 1methylguanine (1-meG) or 3-meC exhibited little such activity. The activity was inhibited by $\mathrm{N}$-oxalylglycine, fumarate and succinate, which were also inhibitors in the 2-OG uncoupled turnover assays. Further study demonstrated that FTO strongly prefers SsDNA as substrates and has no detectable activity toward dsDNA (Gerken et al., 2007; Jia et al., 2008). Similar to some other AlkB members, FTO was later shown to be able to demethylate 3-meU incorporated in ssRNA substrates (Jia et al., 2008). Interestingly, the same study showed that FTO had a little higher enzymatic activity toward 3-meU in ssRAN than 3-meT in ssDNA.

\section{CRYSTAL STRUCTURE OF FTO}

Although FTO was predicted and biochemically confirmed to be an AlkB family DNA/RNA demethylase, it differs from other members in that FTO has a distinct nucleotide specificity, with a strong preference for 3-meT and 3-meU in SSDNA and ssRNA respectively. In addition, FTO contains an extended C terminus that was predicted to have no sequence similarity to any gene with known functions. Therefore the biochemical function of this FTO domain remained completely unknown. Our recent structural and biochemical studies have provided insight into the mechanisms of how FTO protein selects its substrates and how FTO activity is regulated by its C-terminal domain (Han et al., 2010).

Both the $\mathrm{N}$-terminal truncation of 31 residues $(\mathrm{FTO} \Delta 31)$ and existence of the single nucleotide substrate 3-meT were needed to obtain the diffractable crystals of FTO protein. In addition, to prevent the substrate being dissipated, the 2-OG 
analog, $\mathrm{N}$-oxalylglycine (NOG), was used to form a catalytically inert complex. As predicted, the crystal structure of FTO comprises two well-defined domains: an $\mathrm{N}$-terminal domain (residues 32-326, NTD) and a C- terminal domain (residues $327-498$, CTD). The core of the NTD structure is mainly composed of the jelly-roll motif. Consistent with previous prediction, the three highly conserved residues His231, Asp233 and His307 are coordinated to $\mathrm{Fe}(\mathrm{II})$. In addition to chelating $\mathrm{Fe}(\mathrm{II}), \mathrm{NOG}$ also forms salt bonds with Arg316 and Arg322. These structural observations provide further evidence for the notion that NTD of FTO functions as the catalytic domain. The CTD is mainly helical and three of the helices form a three-helix bundle. No known structure was found to have significant similarity to the CTD, indicating that it represents a novel fold.

\section{THE CATALYTIC ROLE OF FTO C-TERMINAL DOMAIN}

In the structure, extensive hydrophobic contacts centering around Phe114 and Cys392 are made between the NTD and the CTD. This structural observation suggests that the CTD plays a role in stabilizing the conformation of the NTD and is therefore important for FTO catalytic activity. Indeed, mutations of these two hydrophobic residues to the charged aspartic acid significantly compromised FTO activity, whereas deletion of the CTD resulted in a catalytically dead FTO. More convincing evidence to support this conclusion came from the observation that co-expression with the CTD rescued the enzymatic activity of NTD via their interaction with each other. Taken together, these results demonstrate the CTD is catalytically inseparable from the NTD.

The mutation I367F that generated a lean phenotype of mice compromised FTO activity. The reason for this was previously proposed to be disruption of FTO homodimerization by this mutation (Church et al., 2009). However, its human equivalent lle370 in the structure FTO is completely buried and unavailable for FTO to form a homodimer. Careful examination of FTO structure revealed that the space surrounding lle 370 is greatly circumscribed by its neighboring residues. Therefore, mutation of this residue to a bulkier one like Phe may perturb the conformation of the CTD, leading to a destabilized NTD and an impaired enzymatic activity. Collectively, the available data (Church et al., 2009; Han et al., 2010) suggest that the CTD plays a role in regulating the activity of FTO. Given that a helix bundle is often involved in protein-protein interaction (Mott and Campbell, 1995), it is tempting to speculate that in vivo the CTD may have some binding partner(s) that can regulate the CTD-NTD interaction. If this is the case, FTO activity would be allosterically regulated.

\section{A UNIQUE SUBSTRATE SELECTION MECHANISM OF FTO}

Interactions of 3-meT with FTO involve both hydrophobic contacts and hydrogen bonds. However, the latter appears to be exclusively responsible for specific recognition of 3-meT by FTO. Both of the two carbonyl oxygen atoms in 3-meT form hydrogen bonds with FTO. Single mutations of the residues in FTO to their equivalents in AlkB (Met61) and hABH2 (Gln112) expected to disrupt these hydrogen bonds resulted in no detectable FTO activity. This observation not just demonstrates the essential role of hydrogen bonds in 3-meT recognition by FTO, but also offers an explanation why other AlkB members are less active toward 3-meT. Further supporting our structural observation, the naturally occurring FTO mutation R96H in human, which is predicted to disrupt the hydrogen bond with $\mathrm{O}^{4}-3-\mathrm{meT}$, resulted in loss of FTO demethylase activity (Meyre et al., 2010). FTO discriminates the subtle difference between 3-meT and 3-meC by making a hydrogen bond with $\mathrm{O}^{4}-3-\mathrm{meT}$ that is absent in the latter. Similarly, lack of $\mathrm{O}^{4}$ or $\mathrm{O}^{2}$ both in 1-meA or 1 -meG makes them poor substrates of FTO. Intriguingly, the mechanism of hydrogen bond-governed substrate selection also exists in the recognition of flipped out uracil by uracil-DNA glycosylase (Slupphaug et al., 1996).

Structural comparison shows the jelly-roll motif of FTO is remarkably similar to those of other AlkB members. One striking structural difference, however, is observed at one outside of the jelly-roll motif. In FTO, an extra loop (referred to L1 loop) completely covers the region defined by $\beta 6, \beta 11, \beta 8$ and $\beta 9$. By contrast, the same region in other AlkB members is exposed and positively charged. This structural observation is fully supported by primary sequence alignment showing that the highly conserved amino acid residues from L1 loop among FTOs are insertions compared to other AlkB members.

In the structures of AlkB- and hABH2-dsDNA complexes, this surface area is involved in binding the unmethylated strand DNA. Structural comparison revealed that L1 loop severely clashes with the unmethylated strand, but not the methylated strand, of the DNA duplex. Thus FTO seems to have evolved a stretch of residues that selects against dsDNA by blocking the unmethylated strand of the DNA duplex from binding the active site of FTO. As far as we know, this is a novel mechanism for a protein to discriminate between ssDNA and dsDNA. This model is consistent with the fact that the structural elements surrounding the conserved jelly-roll motif define different sub-families of 2OG dioxygenases (Hausinger, 2004). Our study provides an example where varied structural elements around the jelly-roll motif within a sub-family can act as a determinant for substrate specificity.

It was previously proposed that Phe102 in hABH2 but absent in $\mathrm{AlkB} / \mathrm{hABH} 3$ can be important in their preferences 
for dsDNA and ssDNA, respectively. Such a mechanism, however, is unable to explain why FTO has no demethylase activity toward dsDNA/RNA. Lack of this residue in AlkB and hABH3 can lower, but not prevent, duplex substrates binding as evidenced by the fact that both of these two proteins are able to repair dsDNA with comparatively lower efficiency.

\section{PERSPECTIVE}

So far the available data have unambiguously established the link between FTO and obesity. However, the molecular mechanism underlying this process remains completely unknown. Clear answers to this question may well take years of active scientific research from diverse fields including biochemistry, genetics, and physiology. An obligated step toward deciphering the molecular mechanisms of how FTO is related to fat mass or obesity would be to identify the in vivo substrates of FTO. The available data (Gerken et al., 2007; Jia et al., 2008; Han et al., 2010) suggest that DNA/RNA, in particular rRNA, may be substrates of FTO in vivo. But it is formally possible that FTO, in addition to DNA/RNA, has other substrates or other enzymatic activity than DNA/RNA demethylation. Coupled with the information from gene expression profiling of the FTO-defective mouse, identification of FTO in vivo substrates would provide clues as to the down stream or up stream components of FTO. Biochemical and structural studies provide reassuring evidence on the significance of FTO catalytic activity, but FTO is an inefficient enzyme in vitro. This raises a possibility that in vivo FTO activity can be regulated by some protein(s). Identification of such protein(s) would be undoubtedly conducive to understanding the acting mechanism of FTO.

One important question regarding FTO as a DNA/RNA demethylase is that whether the catalytic activity is correlated to obesity development. Indirect evidence for this was provided by the observation that a single mutation with an impaired DNA/RNA demethylase activity resulted in lean phenotype of mouse (Church et al., 2009). To further establish such a correlation, transgenic mice with mutations of FTO catalytic residues will be needed. It will be very encouraging to show that these mice generate a similar phenotype to the one carrying the mutation I367F, because this would strongly strengthen the idea that downregulation of FTO catalytic activity can offer a therapeutic approach for treating obesity. From the point view of drug development, a further step would be to verify whether FTO is a valid target for treating obesity or obesity-related diseases. To this end, development of FTOspecific inhibitors is indispensable. Although FTO may have other substrates than DNA/RNA, it is reasonable to assume that their binding is dependent on the known active site of FTO. Therefore, regardless of FTO substrates, FTO-specific inhibitors by targeting its active site will help to verify the druggabiliy of FTO and further clarify if its enzymatic activity is related to obesity development. Although the available data are encouraging, there will be surely a long way to go before obesity can be treated by targeting FTO, even if it turns out to be a valid target in the end.

\section{REFERENCES}

Aas, P.A., Otterlei, M., Falnes, P.O., Vågbø, C.B., Skorpen, F., Akbari, M., Sundheim, O., Bjørås, M., Slupphaug, G., Seeberg, E., et al. (2003). Human and bacterial oxidative demethylases repair alkylation damage in both RNA and DNA. Nature 421, 859-863.

Abelson, P., and Kennedy, D. (2004). The obesity epidemic. Science 304, 1413.

Cecil, J.E., Tavendale, R., Watt, P., Hetherington, M.M., and Palmer, C.N. (2008). An obesity-associated FTO gene variant and increased energy intake in children. N Engl J Med 359, 2558-2566.

Church, C., Lee, S., Bagg, E.A., McTaggart, J.S., Deacon, R., Gerken, T., Lee, A., Moir, L., Mecinović, J., Quwailid, M.M., et al. (2009). A mouse model for the metabolic effects of the human fat mass and obesity associated FTO gene. PLoS Genet 5, e1000599.

Dina, C., Meyre, D., Gallina, S., Durand, E., Körner, A., Jacobson, P., Carlsson, L.M., Kiess, W., Vatin, V., Lecoeur, C., et al. (2007). Variation in FTO contributes to childhood obesity and severe adult obesity. Nat Genet 39, 724-726.

Duncan, T., Trewick, S.C., Koivisto, P., Bates, P.A., Lindahl, T., and Sedgwick, B. (2002). Reversal of DNA alkylation damage by two human dioxygenases. Proc Natl Acad Sci U S A 99, 16660-16665.

Falnes, P.O., Johansen, R.F., and Seeberg, E. (2002). AlkB-mediated oxidative demethylation reverses DNA damage in Escherichia coli. Nature 419, 178-182.

Fischer, J., Koch, L., Emmerling, C., Vierkotten, J., Peters, T., Brüning, J.C., and Rüther, U. (2009). Inactivation of the Fto gene protects from obesity. Nature 458, 894-898.

Frayling, T.M., Timpson, N.J., Weedon, M.N., Zeggini, E., Freathy, R. M., Lindgren, C.M., Perry, J.R., Elliott, K.S., Lango, H., Rayner, N. W., et al. (2007). A common variant in the FTO gene is associated with body mass index and predisposes to childhood and adult obesity. Science 316, 889-894.

Gerken, T., Girard, C.A., Tung, Y.C., Webby, C.J., Saudek, V., Hewitson, K.S., Yeo, G.S., McDonough, M.A., Cunliffe, S., McNeill, L.A., et al. (2007). The obesity-associated FTO gene encodes a 2oxoglutarate-dependent nucleic acid demethylase. Science 318 , 1469-1472.

Groop, L. (2007). From fused toes in mice to human obesity. Nat Genet 39, 706-707.

Han, Z., Niu, T., Chang, J., Lei, X., Zhao, M., Wang, Q., Cheng, W., Wang, J., Feng, Y., and Chai, J. (2010). Crystal structure of the FTO protein reveals basis for its substrate specificity. Nature 464, 1205-1209.

Haupt, A., Thamer, C., Staiger, H., Tschritter, O., Kirchhoff, K., Machicao, F., Häring, H.U., Stefan, N., and Fritsche, A. (2009). Variation in the FTO gene influences food intake but not energy expenditure. Exp Clin Endocrinol Diabetes 117, 194-197.

Hausinger, R.P. (2004). Fell/alpha-ketoglutarate-dependent hydroxylases and related enzymes. Crit Rev Biochem Mol Biol 39, 21-68.

Hinney, A., Nguyen, T.T., Scherag, A., Friedel, S., Brönner, G., Müller, T.D., Grallert, H., Illig, T., Wichmann, H.E., Rief, W., et al. (2007). 
Genome wide association (GWA) study for early onset extreme obesity supports the role of fat mass and obesity associated gene (FTO) variants. PLoS ONE 2, e1361.

Jia, G., Yang, C.G., Yang, S., Jian, X., Yi, C., Zhou, Z., and He, C. (2008). Oxidative demethylation of 3-methylthymine and 3methyluracil in single-stranded DNA and RNA by mouse and human FTO. FEBS Lett 582, 3313-3319.

Kring, S.I., Holst, C., Zimmermann, E., Jess, T., Berentzen, T., Toubro, S., Hansen, T., Astrup, A., Pedersen, O., Sørensen, T.I., et al. (2008). FTO gene associated fatness in relation to body fat distribution and metabolic traits throughout a broad range of fatness. PLoS ONE 3, e2958.

Kurowski, M.A., Bhagwat, A.S., Papaj, G., and Bujnicki, J.M. (2003). Phylogenomic identification of five new human homologs of the DNA repair enzyme AlkB. BMC Genomics 4, 48.

Loenarz, C., and Schofield, C.J. (2008). Expanding chemical biology of 2-oxoglutarate oxygenases. Nat Chem Biol 4, 152-156.

Loos, R.J., and Bouchard, C. (2008). FTO: the first gene contributing to common forms of human obesity. Obes Rev 9, 246-250.

Meyre, D., Proulx, K., Kawagoe-Takaki, H., Vatin, V., GutiérrezAguilar, R., Lyon, D., Ma, M., Choquet, H., Horber, F., Van Hul, W., et al. (2010). Prevalence of loss-of-function FTO mutations in lean and obese individuals. Diabetes 59, 311-318.

Mott, H.R., and Campbell, I.D. (1995). Four-helix bundle growth factors and their receptors: protein-protein interactions. Curr Opin Struct Biol 5, 114-121.

Müller, T.A., Meek, K., and Hausinger, R.P. (2010). Human AlkB homologue $1(\mathrm{ABH} 1)$ exhibits DNA lyase activity at abasic sites. DNA Repair (Amst) 9, 58-65.

Ohashi, J., Naka, I., Kimura, R., Natsuhara, K., Yamauchi, T., Furusawa, T., Nakazawa, M., Ataka, Y., Patarapotikul, J., Nuchnoi, P., et al. (2007). FTO polymorphisms in oceanic populations. J Hum Genet 52, 1031-1035.

Ringvoll, J., Nordstrand, L.M., Vågbø, C.B., Talstad, V., Reite, K., Aas, P.A., Lauritzen, K.H., Liabakk, N.B., Bjørk, A., Doughty, R.W., et al. (2006). Repair deficient mice reveal $m A B H 2$ as the primary oxidative demethylase for repairing $1 \mathrm{meA}$ and $3 \mathrm{meC}$ lesions in DNA. EMBO J 25, 2189-2198.

Sanchez-Pulido, L., and Andrade-Navarro, M.A. (2007). The FTO (fat mass and obesity associated) gene codes for a novel member of the non-heme dioxygenase superfamily. BMC Biochem 8, 23-27.

Scuteri, A., Sanna, S., Chen, W.M., Uda, M., Albai, G., Strait, J., Najjar, S., Nagaraja, R., Orrú, M., Usala, G., et al. (2007). Genomewide association scan shows genetic variants in the FTO gene are associated with obesity-related traits. PLoS Genet 3, e115.

Slupphaug, G., Mol, C.D., Kavli, B., Arvai, A.S., Krokan, H.E., and Tainer, J.A. (1996). A nucleotide-flipping mechanism from the structure of human uracil-DNA glycosylase bound to DNA. Nature 384, 87-92.

Speakman, J.R., Rance, K.A., and Johnstone, A.M. (2008). Polymorphisms of the FTO gene are associated with variation in energy intake, but not energy expenditure. Obesity (Silver Spring) 16, 1961-1965.

Sundheim, O., Vågbø, C.B., Bjørås, M., Sousa, M.M., Talstad, V., Aas, P.A., Drabløs, F., Krokan, H.E., Tainer, J.A., and Slupphaug, G. (2006). Human $A B H 3$ structure and key residues for oxidative demethylation to reverse DNA/RNA damage. EMBO J 25, 3389-3397.

Timpson, N.J., Emmett, P.M., Frayling, T.M., Rogers, I., Hattersley, A. T., McCarthy, M.I., and Davey Smith, G. (2008). The fat mass- and obesity-associated locus and dietary intake in children. Am J Clin Nutr 88, 971-978.

Wardle, J., Carnell, S., Haworth, C.M., Farooqi, I.S., O'Rahilly, S., and Plomin, R. (2008). Obesity associated genetic variation in FTO is associated with diminished satiety. J Clin Endocrinol Metab 93, 3640-3643.

Westbye, M.P., Feyzi, E., Aas, P.A., Vågbø, C.B., Talstad, V.A., Kavli, B., Hagen, L., Sundheim, O., Akbari, M., Liabakk, N.B., et al. (2008). Human AlkB homolog 1 is a mitochondrial protein that demethylates 3-methylcytosine in DNA and RNA. J Biol Chem 283, 25046-25056.

Yang, C.G., Yi, C., Duguid, E.M., Sullivan, C.T., Jian, X., Rice, P.A., and $\mathrm{He}, \mathrm{C}$. (2008). Crystal structures of DNA/RNA repair enzymes AlkB and $A B H 2$ bound to dsDNA. Nature 452, 961-965.

Yu, B., Edstrom, W.C., Benach, J., Hamuro, Y., Weber, P.C., Gibney, B.R., and Hunt, J.F. (2006). Crystal structures of catalytic complexes of the oxidative DNA/RNA repair enzyme AlkB. Nature $439,879-884$. 